\title{
Offline timed pattern matching under uncertainty
}

\author{
Étienne André \\ Université Paris 13, LIPN, CNRS, UMR $7030 \quad$ National Institute of Informatics \\ F-93430, Villetaneuse, France \\ Sokendai (The Graduate University \\ for Advanced Studies) \\ Tokyo, Japan \\ Masaki Waga \\ National Institute of Informatics \\ Tokyo, Japan \\ Sokendai (The Graduate University \\ for Advanced Studies) \\ Kanagawa, Japan \\ JSPS Research Fellow
}

\begin{abstract}
Given a log and a specification, timed pattern matching aims at exhibiting for which start and end dates a specification holds on that log. For example, "a given action is always followed by another action before a given deadline". This problem has strong connections with monitoring realtime systems. We address here timed pattern matching in presence of an uncertain specification, i. e., that may contain timing parameters (e.g., the deadline can be uncertain or unknown). That is, we want to know for which start and end dates, and for what values of the deadline, this property holds. Or what is the minimum or maximum deadline (together with the corresponding start and end dates) for which this property holds. We propose here a framework for timed pattern matching based on parametric timed model checking. In contrast to most parametric timed problems, the solution is effectively computable, and we perform experiments using IMITATOR to show the applicability of our approach.
\end{abstract}

Keywords-monitoring; real-time systems; parametric timed automata;

\section{INTRODUCTION}

Real-time systems are increasingly pervasive in human activities, with systems becoming more and more complex. Monitoring real-time systems consists in deciding whether a $\log$ satisfies a specification. Often, we are rather interested in knowing for which segment of the log the specification is satisfied or violated. This problem can be related to string matching and pattern matching. The timed pattern matching problem was recently formulated in [UFAM14], with subsequent works varying the setting and improving the technique (e.g., [UFAM16], [WAH16], [WHS17]). In [UFAM14], the problem takes as input a timed signal $w$ (values that change over the continuous notion of time) and a timed regular expression $R$ (a real-time extension of regular expressions); and it returns the set of intervals $\left[t, t^{\prime}\right]$ for which the timed regular expression is matched by the log, i. e., $w$ restricted to $\left[t, t^{\prime}\right]$ belongs to the language of $R$.

In [WAH16], [WHS17], we introduced a solution to the timed pattern matching problem where the log is given

This work is partially supported by the ANR national research program PACS (ANR-14-CE28-0002), by JST ERATO HASUO Metamathematics for Systems Design Project (No. JPMJER1603), and by JSPS Grants-in-Aid No. 15KT0012 \& 18J22498. in the form of a timed word (a sequence of events with their associated timestamps), and the specification in the form of a timed automaton (TA), an extension of finite-state automata with clocks [AD94]. In [WAH16], our technique first relied on an extension of the Boyer-Moore algorithm for string matching and its extension to (untimed) pattern matching by Watson and Watson; in [WHS17], it relied on (an automata-theoretic extension of) skip values from the Franek-Jennings-Smyth (FJS) algorithm for string matching [FJS07], so as to improve the efficiency.

As a motivating example, consider the example in Fig. 1. Here $\$$ is a special terminal character. Consider the automaton in Fig. 1a and fix $p_{1}=1$ and $p_{2}=1$-which gives a timed automaton [AD94]. For this timed automaton (say $\mathcal{A}$ ) and the target timed word $w$ in Fig. 1b, the output of the timed pattern matching problem is the set of matching intervals $\left\{\left(t, t^{\prime}\right)|w|_{\left(t, t^{\prime}\right)} \in \mathcal{L}(\mathcal{A})\right\}=\left\{\left(t, t^{\prime}\right) \mid t \in\right.$ $\left.(3.7,3.9), t^{\prime} \in[6.0, \infty)\right\}$.

Contribution: In this work, we consider a more abstract problem: given a (concrete) timed log and an incomplete specification where some of the timing constants may be known with limited precision or completely unknown, what are the intervals and the valuations of the parameters for which the specification holds? Coming back to Fig. 1, the question becomes to exhibit values for $t, t^{\prime}, p_{1}, p_{2}$ for which the specification holds on the log, i. e., $\left\{\left(t, t^{\prime}, v\right)|w|_{\left(t, t^{\prime}\right)} \in\right.$ $\mathcal{L}(v(\mathcal{A}))\}$, where $v$ denotes a valuation of $p_{1}, p_{2}$ and $v(\mathcal{A})$ denotes the replacement of $p_{1}, p_{2}$ in $\mathcal{A}$ with their respective valuation in $v$.

We introduce an approach using as underlying formalisms timed words and parametric timed automata [AHV93], two well-known formalisms in the real-time systems community. We first show that the problem is decidable (which mainly comes from the fact that logs are finite), and we propose a practical solution based on parametric timed model checking.

We implement our method using IMITATOR [AFKS12] and we perform a set of experiments on a set of automotive benchmarks.

Related work: Several algorithms have been proposed for online monitoring of real-time temporal logic spec- 


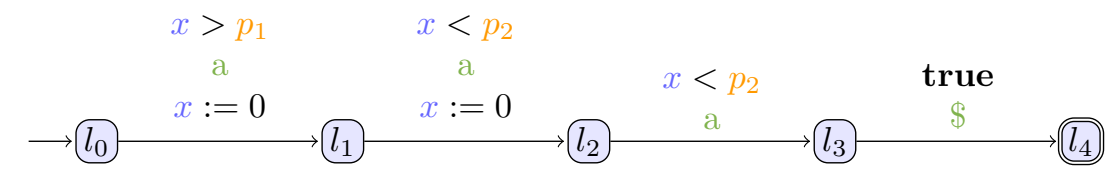

(a) A parametric timed automaton

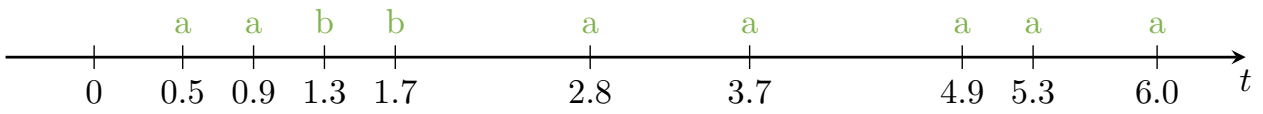

(b) A timed word

Figure 1: An example of parametric timed pattern matching

ifications. Online monitoring consists in monitoring onthe-fly at runtime, while offline monitoring can possibly be performed after the execution is completed, with less hard constraints on the monitoring algorithm performance. An online monitoring algorithm for ptMTL (a past time fragment of MTL [Koy90]) was proposed in [RFB14] and an algorithm for MTL[U,S] (a variant of MTL with both forward and backward temporal modalities) was proposed in [HOW14]. In addition, a case study on an autonomous research vehicle monitoring [KCDK15] shows such procedures can be performed in an actual vehicle.

The approaches most related to ours are [UFAM14], [UFAM16], [Ulu17]. In that series of works, logs are encoded by signals, i. e., values that vary over time. This can be seen as a state-based view, while our timed words are eventbased. The formalism used for specification in [UFAM14], [UFAM16] is timed regular expressions (TREs). An offline monitoring algorithm is presented in [UFAM14] and an online one is in [UFAM16]. These algorithms are implemented in the tool Montre [Ulu17]. The difference between different specification formalisms (TREs, timed automata, temporal logics, etc.) are subtle, but for many realistic examples the difference may not matter.

In [WHS17, we presented an efficient algorithm for online timed pattern matching that employs (an automata-theoretic extension of) skip values from the Franek-Jennings-Smyth (FJS) algorithm for string matching [FJS07]. We showed that our algorithm generally outperforms a brute-force one and our previous BM algorithm [WAH16]: it is twice as fast for some realistic automotive examples. Through our theoretical analysis as well as experiments on memory consumption, our algorithm was shown to be suited for online usage scenarios, too. In comparison, we use here a different approach not based on FJS, but rather on parametric model checking.

Some algorithms have also been proposed for parameter identification of a temporal logic specification with uncertainty over a log. For discrete time setting, an algorithm for an extension of LTL is proposed in [FR08] and for realtime setting, algorithms for parametric signal temporal logic (PSTL) are proposed in [ADMN11], [BFM18]. Although these works are related to our approach, previous approaches do not focus on segments of a log but one whole log. In contrast, we exhibit intervals together with their associated parameter valuations, in a fully symbolic fashion. We believe our matching-based setting is advantageous in many usage scenarios e. g., from hours of a log of a car, extracting timing constraints of a certain actions to cause slipping. Also, our setting allows the patterns with complex timing constraints (see the pattern in Fig. 4c for example).

In [BFMU17], the robust pattern matching problem is considered over signal regular expressions, consisting in computing the quantitative (robust) semantics of a signal relative to an expression. For piecewise-constant and piecewise-linear signals, the problem can be effectively solved using a finite union of zones.

Finally, in an orthogonal direction, in [CJL17], the authors propose a refinement of trace abstractions technique to perform parameter synthesis for real-time systems; they use IMITATOR, as in our work.

A summary of various matching problems is given in Table I

Outline: We first introduce the parametric timed pattern matching problem and the necessary preliminaries in Section II We then introduce our approach based on parametric model checking in Section III. We apply it to benchmarks from [HAF14 in Section IV We finally outline future directions of research in Section V

\section{Preliminaries and objective}

Our target strings are timed words [AD94], that are timestamped words over an alphabet $\Sigma$. Our patterns are given by parametric timed automata [AHV93].

\section{A. Timed words and timed segments}

For an alphabet $\Sigma$, a timed word is a sequence $w$ of pairs $\left(a_{i}, \tau_{i}\right) \in\left(\Sigma \times \mathbb{R}_{\geq 0}\right)$ satisfying $\tau_{i}<\tau_{i+1}$ for any $i \in[1,|w|-1]$. Given a timed word $w$, we often denote it by $(\bar{a}, \bar{\tau})$, where $\bar{a}$ is the sequence $\left(a_{1}, a_{2}, \cdots\right)$ and $\bar{\tau}$ is the sequence $\left(\tau_{1}, \tau_{2}, \cdots\right)$. Let $w=(\bar{a}, \bar{\tau})$ be a timed word. We denote the subsequence $\left(a_{i}, \tau_{i}\right),\left(a_{i+1}, \tau_{i+1}\right), \cdots,\left(a_{j}, \tau_{j}\right)$ by $w(i, j)$. For $t \in \mathbb{R}$ such that $-\tau_{1}<t$, the $t$-shift of $w$ is 


\begin{tabular}{c||c|c|c} 
& log, target & specification, pattern & output \\
\hline \hline string matching & a word $w \in \Sigma^{*}$ & a word pat $\in \Sigma^{*}$ & $\left\{(i, j) \in\left(\mathbb{Z}_{>0}\right)^{2} \mid w(i, j)=\right.$ pat $\}$ \\
\hline pattern matching (PM) & a word $w \in \Sigma^{*}$ & an NFA $\mathcal{A}$ & $\left\{(i, j) \in\left(\mathbb{Z}_{>0}\right)^{2} \mid w(i, j) \in \mathcal{L}(\mathcal{A})\right\}$ \\
\hline timed PM & a timed word $w \in\left(\Sigma \times \mathbb{R}_{>0}\right)^{*}$ & a TA $\mathcal{A}$ & $\left\{\left(t, t^{\prime}\right) \in\left(\mathbb{R}_{>0}\right)^{2}|w|_{\left(t, t^{\prime}\right)} \in \mathcal{L}(\mathcal{A})\right\}$ \\
\hline parametric timed PM & a timed word $w \in\left(\Sigma \times \mathbb{R}_{>0}\right)^{*}$ & a PTA $\mathcal{A}$ & $\left\{\left(t, t^{\prime}, v\right)|w|_{\left(t, t^{\prime}\right)} \in \mathcal{L}(v(\mathcal{A}))\right\}$ \\
\hline
\end{tabular}

Table I: Matching problems

$(\bar{a}, \bar{\tau})+t=(\bar{a}, \bar{\tau}+t)$ where $\bar{\tau}+t=\tau_{1}+t, \tau_{2}+t, \cdots, \tau_{|\tau|}+t$. For timed words $w=(\bar{a}, \bar{\tau})$ and $w^{\prime}=\left(\overline{a^{\prime}}, \overline{\tau^{\prime}}\right)$, their absorbing concatenation is $w \circ w^{\prime}=\left(\bar{a} \circ \overline{a^{\prime}}, \bar{\tau} \circ \overline{\tau^{\prime}}\right)$ where $\bar{a} \circ \overline{a^{\prime}}$ and $\bar{\tau} \circ \overline{\tau^{\prime}}$ are usual concatenations.

For a timed word $w=(\bar{a}, \bar{\tau})$ on $\Sigma$ and $t, t^{\prime} \in \mathbb{R}_{\geq 0}$ satisfying $t<t^{\prime}$, a timed word segment $\left.w\right|_{\left(t, t^{\prime}\right)}$ is defined by the timed word $(w(i, j)-t) \circ\left(\$, t^{\prime}-t\right)$ on the augmented alphabet $\Sigma \sqcup\{\$\}$, where $i, j$ are chosen so that $\tau_{i-1}<t \leq \tau_{i}$ and $\tau_{j} \leq t^{\prime}<\tau_{j+1}{ }^{1}$ Here the fresh symbol $\$$ is called the terminal character.

\section{B. Clocks, parameters and guards}

We assume a set $\mathbb{X}=\left\{x_{1}, \ldots, x_{H}\right\}$ of clocks, i.e., real-valued variables that evolve at the same rate. A clock valuation is a function $\nu: \mathbb{X} \rightarrow \mathbb{R}_{\geq 0}$. We write $\overrightarrow{0}$ for the clock valuation assigning 0 to all clocks. Given $d \in \mathbb{R}_{\geq 0}$, $\nu+d$ denotes the valuation s.t. $(\nu+d)(x)=\nu(x)+d$, for all $x \in \mathbb{X}$. Given $R \subseteq \mathbb{X}$, we define the reset of a valuation $\nu$, denoted by $[\nu]_{R}$, as follows: $[\nu]_{R}(x)=0$ if $x \in R$, and $[\nu]_{R}(x)=\nu(x)$ otherwise.

We assume a set $\mathbb{P}=\left\{p_{1}, \ldots, p_{M}\right\}$ of parameters, i.e., unknown constants. A parameter valuation $v$ is a function $v$ : $\mathbb{P} \rightarrow \mathbb{Q}_{+} 2^{2}$ We assume $\bowtie \in\{<, \leq,=, \geq,>\}$. A guard $g$ is a constraint over $\mathbb{X} \cup \mathbb{P}$ defined by a conjunction of inequalities of the form $x \bowtie d$, or $x \bowtie p$ with $d \in \mathbb{N}$ and $p \in \mathbb{P}$. Given $g$, we write $\nu \models v(g)$ if the expression obtained by replacing each $x$ with $\nu(x)$ and each $p$ with $v(p)$ in $g$ evaluates to true.

\section{Parametric timed automata}

Parametric timed automata (PTA) extend timed automata with parameters within guards and invariants in place of integer constants [AHV93].

Definition 1 (PTA). A PTA $\mathcal{A}$ is a tuple $\mathcal{A}=\left(\Sigma, L, l_{0}, F, \mathbb{X}, \mathbb{P}, I, E\right)$, where:

1) $\Sigma$ is a finite set of actions,

2) $L$ is a finite set of locations,

3) $l_{0} \in L$ is the initial location,

4) $F \subseteq L$ is the set of accepting locations,

5) $\mathbb{X}$ is a finite set of clocks,

6) $\mathbb{P}$ is a finite set of parameters,

7) $I$ is the invariant, assigning to every $l \in L$ a guard $I(l)$,

\footnotetext{
${ }^{1}$ Observe that, in contrast with [WAH16, WHS17, we use here closed intervals, i. e., $t, t^{\prime}$ may be equal to the start or end time of the word.

${ }^{2}$ We choose $\mathbb{Q}_{+}$by consistency with most of the PTA literature, but also because, for classical PTAs, choosing $\mathbb{R}_{\geq 0}$ leads to undecidability [Mil00].
}

8) $E$ is a finite set of edges $e=\left(l, g, a, R, l^{\prime}\right)$ where $l, l^{\prime} \in$ $L$ are the source and target locations, $a \in \Sigma, R \subseteq \mathbb{X}$ is a set of clocks to be reset, and $g$ is a guard.

Given a parameter valuation $v$, we denote by $v(\mathcal{A})$ the non-parametric structure where all occurrences of a parameter $p_{i}$ have been replaced by $v\left(p_{i}\right)$. We denote as a timed automaton any structure $v(\mathcal{A})$, by assuming a rescaling of the constants: by multiplying all constants in $v(\mathcal{A})$ by their least common denominator, we obtain an equivalent (integervalued) TA, as defined in [AD94.

The synchronous product (using strong broadcast, i.e., synchronization on shared actions) of several PTAs gives a PTA.

Definition 2 (synchronized product of PTAs). Let $N \in \mathbb{N}$. Given a set of PTAs $\mathcal{A}_{i}=\left(\Sigma_{i}, L_{i},\left(l_{0}\right)_{i}, F_{i}, \mathbb{X}_{i}, \mathbb{P}_{i}, I_{i}, E_{i}\right)$, $1 \leq i \leq N$, the synchronized product of $\mathcal{A}_{i}, 1 \leq i \leq$ $N$, denoted by $\mathcal{A}_{1}\left\|\mathcal{A}_{2}\right\| \cdots \| \mathcal{A}_{N}$, is the tuple $\left(\Sigma, L, l_{0}, F, \mathbb{X}, \mathbb{P}, I, E\right)$, where:

1) $\Sigma=\bigcup_{i \overline{\bar{N}} 1}^{N} \Sigma_{i}$,

2) $L=\prod_{i=1}^{\bar{N}} L_{i}$

3) $l_{0}=\left(\left(l_{0}\right)_{1}, \ldots,\left(l_{0}\right)_{N}\right)$,

4) $F=\left\{\left(l_{1}, \ldots, l_{N}\right) \in L \mid \exists i \in[1, N]\right.$ s.t. $\left.l_{i} \in F_{i}\right\}$,

5) $\mathbb{X}=\bigcup_{1 \leq i \leq N} \mathbb{X}_{i}$,

6) $\mathbb{P}=\bigcup_{1 \leq i \leq N} \mathbb{P}_{i}$,

7) $I\left(\left(l_{1}, \ldots, l_{N}\right)\right)=\bigwedge_{i=1}^{N} I_{i}\left(l_{i}\right)$ for all $\left(l_{1}, \ldots, l_{N}\right) \in L$, and $E$ is defined as follows. For all $a \in \Sigma$, let $\zeta_{a}$ be the subset of indices $i \in 1, \ldots, N$ such that $a \in \Sigma_{i}$. For all $a \in \Sigma$, for all $\left(l_{1}, \ldots, l_{N}\right) \in L$, for all $\left(l_{1}^{\prime}, \ldots, l_{N}^{\prime}\right) \in L$, $\left(\left(l_{1}, \ldots, l_{N}\right), g, a, R,\left(l_{1}^{\prime}, \ldots, l_{N}^{\prime}\right)\right) \in E$ if:

- for all $i \in \zeta_{a}$, there exist $g_{i}, R_{i}$ such that $\left(l_{i}, g_{i}, a, R_{i}, l_{i}^{\prime}\right) \in E_{i}, g=\bigwedge_{i \in \zeta_{a}} g_{i}, R=\bigcup_{i \in \zeta_{a}} R_{i}$, and,

- for all $i \notin \zeta_{a}, l_{i}^{\prime}=l_{i}$.

Let us now recall the concrete semantics of TA.

Definition 3 (Semantics of a TA). Given a PTA $\mathcal{A}=$ $\left(\Sigma, L, l_{0}, F, \mathbb{X}, \mathbb{P}, I, E\right)$, and a parameter valuation $v$, the semantics of $v(\mathcal{A})$ is given by the timed transition system (TTS) $\left(S, s_{0}, \rightarrow\right)$, with

- $S=\left\{(l, \nu) \in L \times \mathbb{R}_{\geq 0}^{H} \mid \nu \models v(I(l))\right\}$,

- $s_{0}=\left(l_{0}, \overrightarrow{0}\right)$,

- $\rightarrow$ consists of the discrete and (continuous) delay transition relations:

1) discrete transitions: $(l, \nu) \quad \stackrel{e}{\mapsto} \quad\left(l^{\prime}, \nu^{\prime}\right)$, if $(l, \nu),\left(l^{\prime}, \nu^{\prime}\right) \in S, \quad$ and there exists 
$e=\left(l, g, a, R, l^{\prime}\right) \in E$, such that $\nu^{\prime}=[\nu]_{R}$, and $\nu \models v(g)$.

2) delay transitions: $(l, \nu) \stackrel{d}{\mapsto}(l, \nu+d)$, with $d \in \mathbb{R}_{\geq 0}$, if $\forall d^{\prime} \in[0, d],\left(l, \nu+d^{\prime}\right) \in S$.

Moreover we write $(l, \nu) \stackrel{(e, d)}{\longrightarrow}\left(l^{\prime}, \nu^{\prime}\right)$ for a combination of a delay and discrete transition if $\exists \nu^{\prime \prime}:(l, \nu) \stackrel{d}{\mapsto}\left(l, \nu^{\prime \prime}\right) \stackrel{e}{\mapsto}$ $\left(l^{\prime}, \nu^{\prime}\right)$.

Given a TA $v(\mathcal{A})$ with concrete semantics $\left(S, s_{0}, \rightarrow\right)$, we refer to the states of $S$ as the concrete states of $v(\mathcal{A})$. A run of $v(\mathcal{A})$ is a possibly infinite alternating sequence of concrete states of $v(\mathcal{A})$ and pairs of edges and delays starting from the initial state $s_{0}$ of the form $s_{0},\left(e_{0}, d_{0}\right), s_{1}, \cdots$ with $i=0,1, \ldots, e_{i} \in E, d_{i} \in \mathbb{R}_{\geq 0}$ and $\left(s_{i}, e_{i}, s_{i+1}\right) \in \rightarrow$. Given such a run, the associated timed word is $\left(a_{1}, \tau_{1}\right),\left(a_{2}, \tau_{2}\right), \cdots$, where $a_{i}$ is the action of edge $e_{i-1}$, and $\tau_{i}=\sum_{0<j<i-1} d_{j}$, for $i=1,2 \ldots \beta^{3}$ Given a state $s=(l, \nu)$, we say that $s$ is reachable in $v(\mathcal{A})$ if $s$ appears in a run of $v(\mathcal{A})$. By extension, we say that $l$ is reachable; and by extension again, given a set $T$ of locations, we say that $T$ is reachable if there exists $l \in T$ such that $l$ is reachable in $v(\mathcal{A})$.

A finite run is accepting if its last state $(l, \nu)$ is such that $l \in F$. The (timed) language $\mathcal{L}(v(\mathcal{A}))$ is defined to be the set of timed words associated with all accepting runs of $v(\mathcal{A})$.

\section{Reachability synthesis}

We will use here reachability synthesis to solve parametric timed pattern matching. This procedure, called EFsynth, takes as input a PTA $\mathcal{A}$ and a set of target locations $T$, and attempts to synthesize all parameter valuations $v$ for which $T$ is reachable in $v(\mathcal{A})$. EFsynth was formalized in e.g., [JLR15] and is a procedure that may not terminate, but that computes an exact result (sound and complete) if it terminates. EFsynth traverses the parametric zone graph of $\mathcal{A}$, which is a potentially infinite extension of the wellknown zone graph of TAs (see, e.g., [ACEF09], [JLR15] for a formal definition).

\section{E. Timed pattern matching}

Let us recall timed pattern matching [WAH16], [WHS17].

\section{Timed pattern matching problem:}

INPUT: a TA $\mathcal{A}$, a timed word $w$ over a common alphabet $\Sigma$

PROBLEM: compute all the intervals $\left(t, t^{\prime}\right)$ for which the segment $\left.w\right|_{\left(t, t^{\prime}\right)}$ is accepted by $\mathcal{A}$. That is, it requires the match set $\mathcal{M}(w, \mathcal{A})=\left\{\left(t, t^{\prime}\right)|w|_{\left(t, t^{\prime}\right)} \in \mathcal{L}(\mathcal{A})\right\}$.

The match set $\mathcal{M}(w, \mathcal{A})$ is in general uncountable; however it allows finite representation, as a finite union of special polyhedra called zones (see [BY03], [WAH16]).

\footnotetext{
${ }^{3}$ The " -1 " in indices comes from the fact that, following usual conventions in the literature, states are numbered starting from 0 while words are numbered from 1 .
}

We now extend this problem to parameters by allowing a specification expressed using PTAs. The problem now requires not only the start and end dates for which the property holds, but also the associated parameter valuations.

\section{Parametric timed pattern matching problem:}

INPUT: a PTA $\mathcal{A}$, a timed word $w$ over a common alphabet $\Sigma$

PROBLEM: compute all the triples $\left(t, t^{\prime}, v\right)$ for which the segment $\left.w\right|_{\left(t, t^{\prime}\right)}$ is accepted by $v(\mathcal{A})$. That is, it requires the match set $\mathcal{M}(w, \mathcal{A})=\left\{\left(t, t^{\prime}, v\right)|w|_{\left(t, t^{\prime}\right)} \in\right.$ $\mathcal{L}(v(\mathcal{A}))\}$.

We will see that this match set can still be represented as a finite union of polyhedra, but in more dimensions, viz., $|\mathbb{P}|+2$, i. e., the number of parameters +2 further dimensions for $t$ and $t^{\prime}$. However, the form of the obtained polyhedra is more general than zones, as parameters may "accumulate" to produce sums of parameters with coefficients (e.g., $3 \times p_{1}<$ $\left.p_{2}+2 \times p_{3}\right)$.

\section{TIMED PATTERN MATCHING UNDER UNCERTAINTY}

\section{A. General approach}

We make the following two assumptions, that do not impact the correctness of our method, but simplify the subsequent reasoning.

Assumption 1. As in [WAH16], [WHS17], we assume that the pattern automaton contains a single final location, and that all transitions to this final location are labeled with $\$$.

Assumption 2. We assume that the pattern automaton contains no action not part of the timed word alphabet, with the exception of the special action $\$$.

Both assumptions are easy to remove in practice: for Assumption 1, if the pattern PTA contains more than one final location, they can be merged into a single final location. For Assumption 2, the transitions labeled with actions not part of the timed word alphabet can simply be deleted.

We show using the following approach that parametric timed pattern matching can reduce to parametric reachability analysis.

1) We turn the pattern into a symbolic pattern, by allowing it to start anytime. In addition, we use two parameters to measure the (symbolic) starting time and the (symbolic) ending time of the pattern.

2) We turn the timed word into a (non-parametric) timed automaton, that uses a single clock $x_{a b s}$, that measures the absolute time.

3) We consider the synchronized product of the symbolic pattern PTA and the timed word (P)TA.

4) We run the reachability synthesis algorithm to derive all possible parameter valuations for which the final location of the pattern automaton is reachable. 


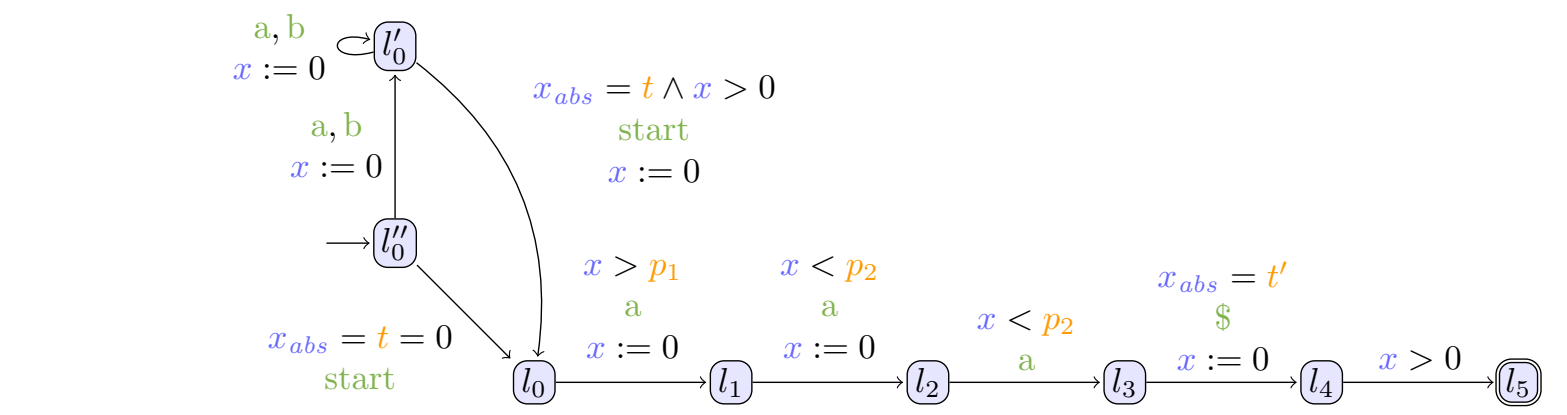

(a) MakeSymbolic applied to the PTA in Fig. 1a

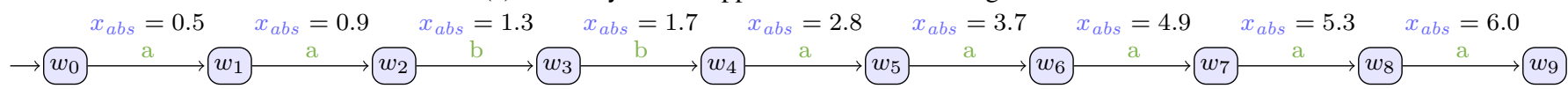

(b) TW2PTA applied to the timed word in Fig. 1b

Figure 2: Our transformations exemplified on Fig. 1

\section{B. Our approach step by step}

1) Making the pattern symbolic: In this first step, we first add two parameters $t$ and $t^{\prime}$, which encode the (symbolic) start and end time where the pattern holds on the input timed word. This way, we will obtain a result in the form of a finite union of polyhedra in $M+2$ dimensions, where the 2 additional dimensions come from the addition of $t$ and $t^{\prime}$. We also add a clock $x_{a b s}$ initially 0 and never reset (this clock is shared by the pattern PTA and the subsequent timed word TA). Then, we modify the pattern PTA as follows:

1) we add two fresh locations ( $\operatorname{say} l_{0}^{\prime}$ and $l_{0}^{\prime \prime}$ ) prior to the initial location $l_{0}$

2) we add a fresh clock (say $x$ ); in practice, as this clock is used only in the initial location, an existing clock of the pattern may be reused;

3) we add an unguarded self-loop allowing any action of the timed word on $l_{0}^{\prime}$, and resetting $x$;

4) we add an unguarded transition from $l_{0}^{\prime \prime}$ to $l_{0}^{\prime}$ allowing any action of the timed word on $l_{0}^{\prime}$, and resetting $x$;

5) we add a transition from $l_{0}^{\prime}$ to $l_{0}$ guarded by $x_{a b s}=$ $t \wedge x>0$, labeled with a fresh action start and resetting all clocks of the pattern (except $x_{a b s}$ );

6) we add a transition from $l_{0}^{\prime \prime}$ to $l_{0}$ guarded by $x_{a b s}=$ $t \wedge x_{a b s}=0$, labeled with start;

7) we add a guard $x_{a b s}=t^{\prime}$ and reset $x$ on the final transitions labeled with $\$$;

8) we add an extra location after the former final location, with a transition guarded by $x>0$;

9) the initial location of the modified PTA becomes $l_{0}^{\prime \prime}$.

Let us give the intuition behind our transformation. First, the two guards $x_{a b s}=t$ and $x_{a b s}=t^{\prime}$ allow to record symbolically the value of the starting and ending dates. Second, the new locations $l_{0}^{\prime \prime}$ and $l_{0}^{\prime}$ allow the pattern to "start anytime"; that is, it can synchronize with the timed word TA for an arbitrary long time while staying in the initial location $l_{0}^{\prime \prime}$ (and therefore not matching the pattern), and start (using the transition from $l_{0}^{\prime}$ to $l_{0}$ ) anytime. Third, due to the constraint $x>0$, a non-zero time must elapse between the last action before the pattern start and the actual pattern start. The distinction between $l_{0}^{\prime \prime}$ and $l_{0}^{\prime}$ is necessary to also allow starting the pattern at $x_{a b s}=0$ if no action occurred before. Finally, the guard $x>0$ just before the final location ensures the next action of the system (if any) is taken after a non-zero delay, following our definitions of timed word and projection.

Let MakeSymbolic denote this procedure.

Consider the pattern PTA $\mathcal{A}$ in Fig. 1a. The result of MakeSymbolic $(\mathcal{A})$ is given in Fig. 2a. Note that we use the same clock $x$ for both the extra clock introduced by our construction and the original clock of the pattern automaton from Fig. 1a

2) Converting the timed word into a $(P) T A$ : In this second step, we convert the timed word into a (nonparametric) timed automaton. This is very straightforward, and simply consists in converting a timed word of the form $\left(a_{1}, \tau_{1}\right), \cdots,\left(a_{n}, \tau_{n}\right)$ into a sequence of transitions labeled with $a_{i}$ and guarded with $x_{a b s}=\tau_{i}$ (recall that $x_{a b s}$ measures the absolute time and is shared by the timed word automaton and the pattern automaton).

Let TW2PTA denote this procedure.

Consider the timed word $w$ in Fig. 1b The result of $\operatorname{TW2PTA}(w)$ is given in Fig. 2b

3) Synchronized product: The last part of the method consists in performing the synchronized product of MakeSymbolic $(\mathcal{A})$ and $\operatorname{TW} 2 \operatorname{PTA}(w)$, and calling EFsynth on the resulting PTA.

We summarize our method $\operatorname{PTPM}(\mathcal{A}, w)$ in $\operatorname{Algorithm~} 1$

Example 1. Consider again the timed word $w$ and the PTA pattern $\mathcal{A}$ in Fig. 1. The result of $\operatorname{PTPM}(\mathcal{A}, w)$ is as follows: 


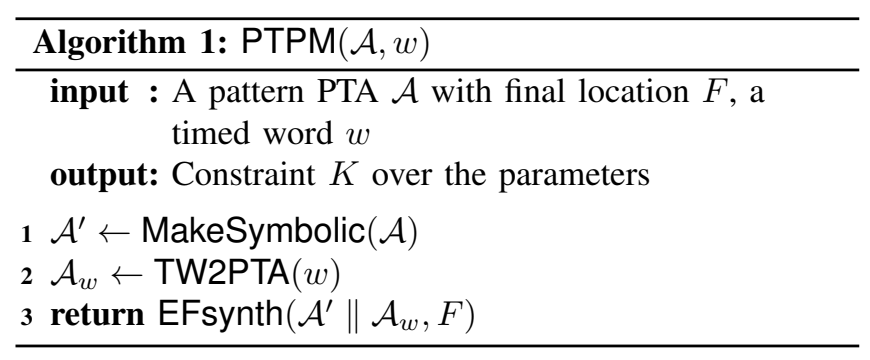

$$
\begin{aligned}
& 1.7<t<2.8-p_{1} \wedge 4.9 \leq t^{\prime}<5.3 \wedge p_{2}>1.2 \\
& \vee \quad 2.8<t<3.7-p_{1} \wedge 5.3 \leq t^{\prime}<6 \wedge p_{2}>1.2 \\
& \vee 3.7<t<4.9-p_{1} \wedge t^{\prime} \geq 6 \wedge p_{2}>0.7
\end{aligned}
$$

Observe that, for the parameter valuation given in the introduction $\left(p_{1}=p_{2}=1\right)$, only the pattern corresponding to the last disjunct could be obtained, i. e., the pattern that matches the last three $a$ of the timed word in Fig. 1b. In contrast, the first disjunct can match the first three $a$ coming after the two $b s$, while the second disjunct allows to match the three $a$ s in the middle of the last five $a$ s in Fig. 1b.

We give various projections of this constraint onto two dimensions in Fig. 3 (the difference between plain red and light red is not significant-light red constraints denote unbounded constraints towards at least one dimension).

\section{Termination}

We state below the termination of our procedure.

Lemma 1 (termination). Let $\mathcal{A}$ be a PTA encoding a parametric pattern, and $w$ be a timed word. Then $\operatorname{PTPM}(\mathcal{A}, w)$ terminates.

Proof: First, observe that there may be non-determinism in the pattern PTA, i.e., the timed word can potentially synchronize with two transitions labeled with the same action from a given location. Even if there is no syntactic nondeterminism, nondeterminism can appear due to the interleaving of the initial start action: in Fig. 2, the first a of the timed word can either synchronize with the selfloop on $l_{0}^{\prime}$, or the start action can first occur, and then the first a synchronizes of the timed word with the a labeling the transition from $l_{0}$ to $l_{1}$ of the pattern PTA. Second, the pattern PTA may well have loops (and this is the case in our experiments in Section IV-C, which yields an infinite parametric zone graph (for the pattern automaton not synchronized with the word automaton). However, let us show that only a finite part of the parametric zone graph is explored by EFsynth: indeed, since TW2PTA $(w)$ is only a finite sequence, and thanks to the strong synchronization between the pattern PTA and the timed word PTA and due to Assumption 2, only a finite number of finite discrete paths in the synchronized product will be explored. The only interleaving is due to the initial start action (which appears twice in the pattern PTA but can only be taken once at most due to the mutually exclusive guards $x=0$ and $x>0$ ), and due to the final $\$$ action, that only appear on the last transition to the last-but-one final location. As the pattern PTA is finitely branching, this gives a finite number of finite paths. The length of each path is clearly bounded by $|w|+3$. Let us now consider the maximal number of such paths: given a location in $\operatorname{TW} 2 \mathrm{PTA}(w)$, the choice of the action (say a) is entirely deterministic. However, the pattern PTA may be non-deterministic, and can synchronize with $B$ outgoing transitions labeled with a, which gives $B^{|w|}$ combinations. In addition, the start action can be inserted exactly once, at any position in the timed word (from before the first action to after the last action of the word-in the case of an empty pattern): this gives therefore $(|w|+1) \times B^{|w|}$ different runs. (The $\$$ is necessarily the last-but-one action, and does not impact the number of runs, as the (potential) outgoing transitions from the final location are not explored.) Altogether, a total number of at most $(|w|+3) \times(|w|+1) \times B^{|w|}$ symbolic states is explored by EFsynth in the worst case.

Lemma 1 may not come as a surprise, as the input timed word is finite. But it is worth noting that it comes in contrast with the fact that the wide majority of decision problems are undecidable for parametric timed automata, including the emptiness of the valuation set for which a given location is reachable both, for integer- and rationalvalued parameters [AHV93], [Mil00] (see [And17] for a survey).

\section{Pattern matching with optimization}

We also address the following optimization problem: given a timed word and a pattern containing parameters, what is the minimum or maximum value of a given parameter such that the pattern is matched by the timed word? That is, we are only interested in the optimal value of the given parameter, and not in the full list of matches as in PTPM.

While this problem can be solved using our solution from Section III-A (by computing the multidimensional constraint, and then eliminating all parameters but the target parameter, using variable elimination techniques), we use here a dedicated approach, with the hope it be more efficient. Instead of managing all symbolic matches (i.e., a finite union of polyhedra), we simply manage the current optimum; in addition, we cut branches that cannot improve the optimum, with the hope to reduce the number of states explored. For example, assume parameter $p$ is to be minimized; if the current minimum is $p>2$, and if a branch is such that $p \geq 3$, then this branch will not improve the

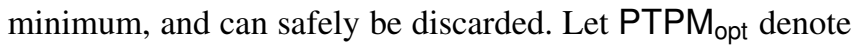
this procedure.

Example 2. Consider again the timed word $w$ and the PTA pattern $\mathcal{A}$ in Fig. 1 Minimizing $p_{2}$ so that the pattern 


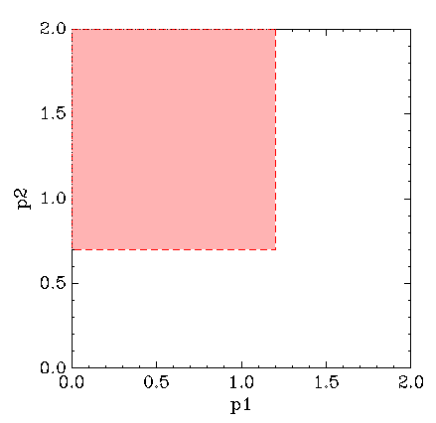

(a) On $p_{1}$ and $p_{2}$

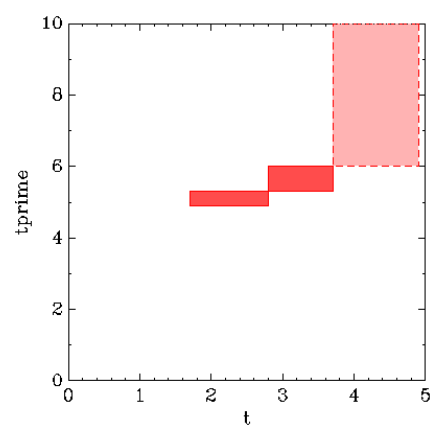

(b) On $t$ and $t^{\prime}$

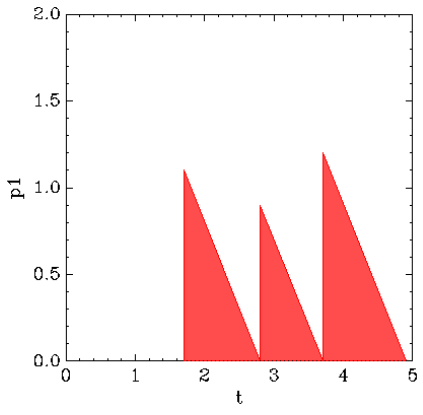

(c) On $t$ and $p_{1}$

Figure 3: Projections of the result of parametric timed pattern matching on Fig. 1

matches the timed word for at least one position gives $p_{2}>0.7$, while maximizing $p_{1}$ gives $p_{1}<1.2$.

\section{EXPERIMENTS}

We evaluated our approach against two standard benchmarks from [HAF14], already used in [WHS17], as well as a third benchmark specifically designed to test the limits of parametric timed pattern matching. We fixed no bounds for our parameters.

We used IMITATOR AFKS12] to perform the parameter synthesis (algorithm EFsynth). IMITATOR relies on the Parma Polyhedra Library (PPL) [BHZ08] to compute symbolic states. It was shown in [BFMU17] that polyhedra may be dozens of times slower than more efficient data structures such as DBMs (difference bound matrices); however, for parametric analyses, DBMs are not suitable, and parameterized extensions (e.g., in [HRSV02]) still need polyhedra in their representation.

We used a slightly modified version of IMITATOR for technical reasons (see Appendix A). We wrote a simple Python script to implement the TW2PTA procedure; the patterns (Fig. 4) were manually transformed following the MakeSymbolic procedure, and converted into the input language of IMITATOR.

We ran experiments using IMITATOR 2.10.4 "Butter Jellyfish" on a Dell Precision 3620 i7-7700 $3.60 \mathrm{GHz}$ with $64 \mathrm{GiB}$ memory running Linux Mint 19 beta 64 bits. Sources, binaries, models, logs can be found at WWW. imitator.fr/static/ICECCS18.

\section{A. GEAR}

Benchmark GEAR is inspired by the scenario of monitoring the gear change of an automatic transmission system. We conducted simulation of the model of an automatic transmission system [HAF14]. We used S-TaLiRo [ALFS11] to generate an input to this model; it generates a gear change signal that is fed to the model. A gear is chosen from $\left\{g_{1}, g_{2}, g_{3}, g_{4}\right\}$. The generated gear change is recorded in a timed word. The set $W$ consists of 10 timed words; the length of each word is 1,467 to 14,657 .
The pattern PTA $\mathcal{A}$, shown in Fig. 4a detects the violation of the following condition: If the gear is changed to 1 , it should not be changed to 2 within $p$ seconds. This condition is related to the requirement $\phi_{5}^{A T}$ proposed in [HAF14] (the nominal value for $p$ in [HAF14] is 2).

We tabulate our experiments in Table II. We give from left to right the length of the timed word in terms of actions and time, then the data for PTPM (the number of symbolic states explored, the number of (symbolic) matches found, the parsing time and the computation time excluding parsing)

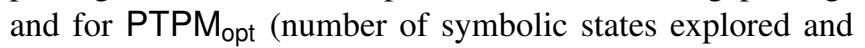
computation time) using IMITATOR. The parsing time for PTPM $_{\text {opt }}$ is almost identical to PTPM and is therefore omitted.

The corresponding chart is given in Fig. 5a (PTPM is given in plain black, and PTPM ${ }_{\text {opt }}$ in red dashed). PTPM ${ }_{\text {opt }}$ brings a gain in terms of memory (symbolic states) of about $25 \%$, while the gain in time is about $20 \%$.

\section{B. ACCEL}

The $W$ of benchmark ACCEL is also constructed from the Simulink model of the automated transmission system [HAF14]. For this benchmark, the (discretized) value of three state variables are recorded in $W$ : engine RPM (discretized to "high" and "low" with a certain threshold), velocity (discretized to "high" and "low" with a certain threshold), and 4 gear positions. We used S-TaLiRo [ALFS11] to generate a input sequence of gear change. Our set $W$ consists of 10 timed words; the length of each word is 2,559 to 25,137 .

The pattern PTA $\mathcal{A}$ of this benchmark is shown in Fig. 4b This pattern matches a part of a timed word that violates the following condition: If a gear changes from 1 to 2,3 , and 4 in this order in $p$ seconds and engine RPM becomes large during this gear change, then the velocity of the car must be sufficiently large in one second. This condition models the requirement $\phi_{8}^{A T}$ proposed in [HAF14] (the nominal value for $p$ in [HAF14] is 10).

Experiments are tabulated in Table III The corresponding chart is given in Fig. 5b This time, PTPM opt $_{\text {brings almost }}$ 


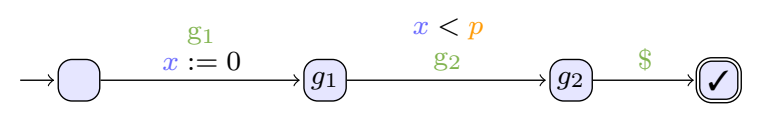

(a) GEAR

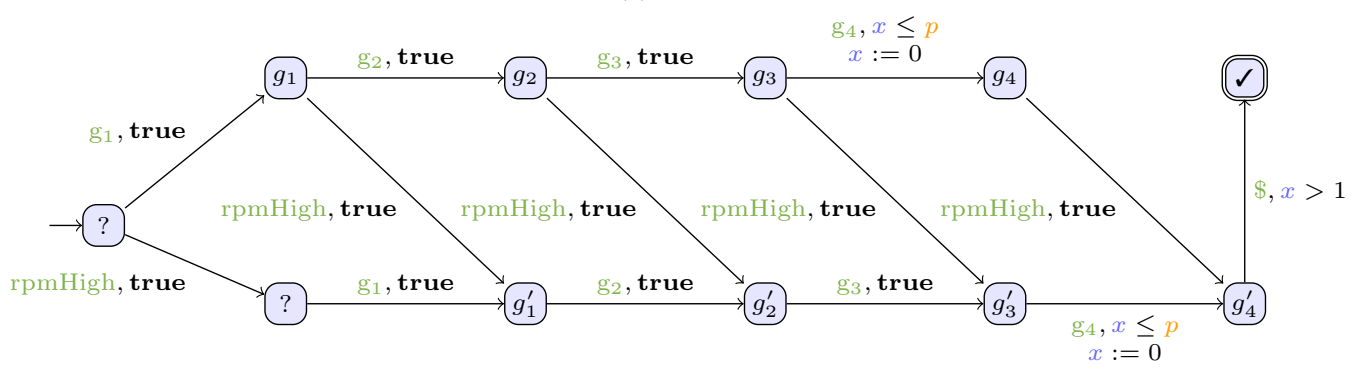

(b) ACCEL

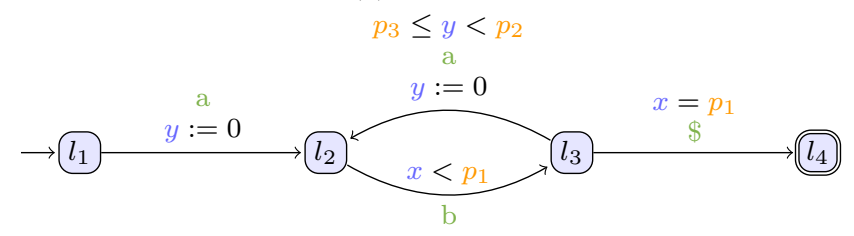

(c) BLOWUP

Figure 4: Experiments: patterns

\begin{tabular}{|c|c|c|c|c|c|c|c|}
\hline \multicolumn{2}{|c|}{ Model } & \multicolumn{4}{|c|}{ PTPM } & \multicolumn{2}{|c|}{ PTPM $_{\text {opt }}$} \\
\hline Length & Time frame & States & Matches & Parsing (s) & Comp. (s) & States & Comp. (s) \\
\hline 1,467 & 1,000 & 4,453 & 379 & 0.02 & 1.60 & 3,322 & 0.94 \\
\hline 2,837 & 2,000 & 8,633 & 739 & 0.33 & 2.14 & 6,422 & 1.70 \\
\hline 4,595 & 3,000 & 14,181 & 1,247 & 0.77 & 3.63 & 10,448 & 2.85 \\
\hline 5,839 & 4,000 & 17,865 & 1,546 & 1.23 & 4.68 & 13,233 & 3.74 \\
\hline 7,301 & 5,000 & 22,501 & 1,974 & 1.94 & 5.88 & 16,585 & 4.79 \\
\hline 8,995 & 6,000 & 27,609 & 2,404 & 2.96 & 7.28 & 20,413 & 5.76 \\
\hline 10,316 & 7,000 & 31,753 & 2,780 & 4.00 & 8.38 & 23,419 & 6.86 \\
\hline 11,831 & 8,000 & 36,301 & 3,159 & 5.39 & 9.75 & 26,832 & 7.87 \\
\hline 13,183 & 9,000 & 40,025 & 3,414 & 6.86 & 10.89 & 29,791 & 8.61 \\
\hline 14,657 & 10,000 & 44,581 & 3,816 & 8.70 & 12.15 & 33,141 & 9.89 \\
\hline
\end{tabular}

Table II: Experiments: GEAR

no gain in terms of states, and a loss of speed of about 15 to $20 \%$, which may come from the additional polyhedra inclusion checks to test whether a branch is less good than the current optimum.

\section{BLOWuP}

As a third experiment, we considered an original (toy) benchmark that acts as a worst case situation for parametric timed pattern matching. Consider the PTA pattern in Fig. 4c and assume a timed word consisting in an alternating sequence of "a" and "b". Observe that the time from the pattern beginning (that resets $x$ ) to the end is exactly $p_{1}$ time units. Also observe that the duration of the loop through $l_{2}$ and $l_{3}$ has a duration in $\left[p_{3}, p_{2}\right)$; therefore, for values sufficiently small of $p_{2}, p_{3}$, one can always match a larger number of loops. That is, for a timed word of length $2 n$ alternating between "a" and "b", there will be $n$ possible matches from position 0 (with $n$ different parameter constraints), $n-1$ from position 1 , and so on, giving a total number of $\frac{n(n+1)}{2}$ matches with different constraints in 5 dimensions.

Note that this worst case situation is not specific to our approach, but would appear independently of the approach chosen for parametric timed pattern matching.

We generated random timed words of various sizes, all alternating exactly between "a" and "b". Our set $W$ consists of 5 timed words of length from 200 to 1,000 .

Experiments are tabulated in Table IV] The corresponding chart is given in Fig. 5c. PTPM becomes clearly non-linear

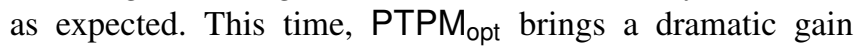
in both memory and time; even more interesting, PTPM opt $_{1}$ remains perfectly linear.

\section{Discussion}

A first positive outcome is that our method is effectively able to perform parametric pattern matching on words of length up to several dozens of thousands, and is able to output results in the form of several dozens of thousands 


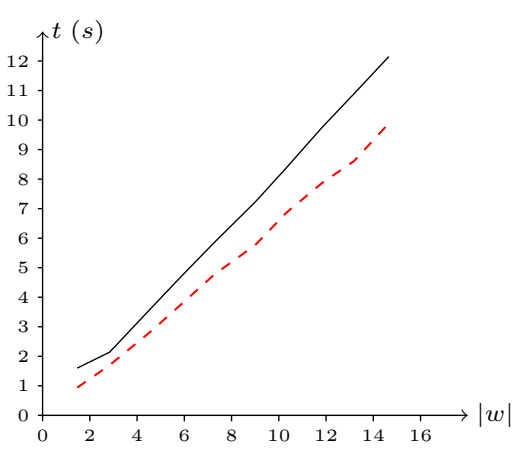

(a) GEAR

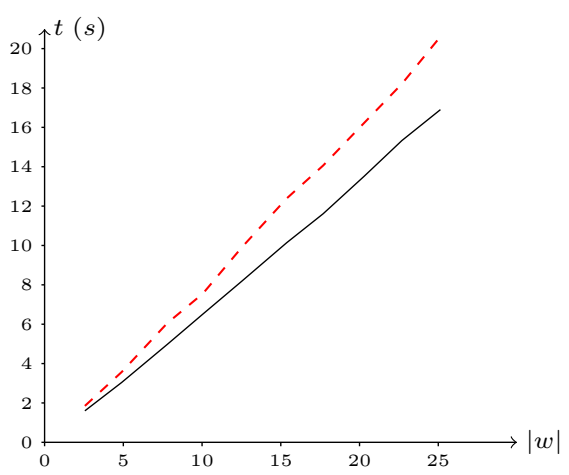

(b) ACCEL

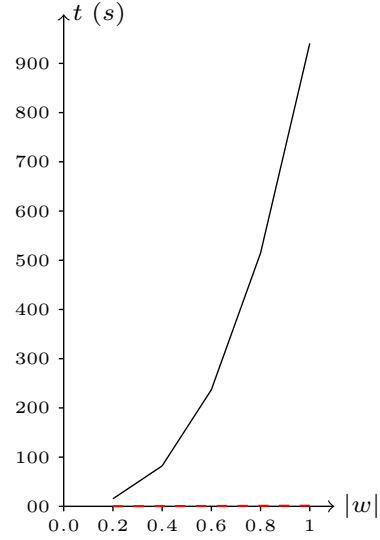

(c) BLOWUP

Figure 5: Experiments: charts $(x$-scale $\times 1,000)$

\begin{tabular}{|r|r|r|r|r|r|r|r|}
\hline \multicolumn{2}{|c|}{ Model } & \multicolumn{3}{c|}{ PTPM } & \multicolumn{2}{c|}{ PTPM opt } \\
Length & Time frame & States & Matches & Parsing (s) & Comp. (s) & States & Comp. (s) \\
\hline 2,559 & 1,000 & 6,504 & 2 & 0.27 & 1.60 & 6,502 & 1.85 \\
\hline 4,894 & 2,000 & 12,429 & 2 & 0.86 & 3.04 & 12,426 & 3.57 \\
\hline 7,799 & 3,000 & 19,922 & 7 & 2.21 & 4.98 & 19,908 & 6.06 \\
\hline 10,045 & 4,000 & 25,520 & 3 & 3.74 & 6.51 & 25,514 & 7.55 \\
\hline 12,531 & 5,000 & 31,951 & 9 & 6.01 & 8.19 & 31,926 & 9.91 \\
\hline 15,375 & 6,000 & 39,152 & 7 & 9.68 & 10.14 & 39,129 & 12.39 \\
\hline 17,688 & 7,000 & 45,065 & 9 & 13.40 & 11.61 & 45,039 & 14.06 \\
\hline 20,299 & 8,000 & 51,660 & 10 & 18.45 & 13.52 & 51,629 & 16.23 \\
\hline 22,691 & 9,000 & 57,534 & 11 & 24.33 & 15.33 & 57,506 & 18.21 \\
\hline 25,137 & 10,000 & 63,773 & 13 & 31.35 & 16.90 & 63,739 & 20.61 \\
\hline
\end{tabular}

Table III: Experiments: ACCEL

\begin{tabular}{|r|r|r|r|r|r|r|r|}
\hline \multicolumn{3}{|c|}{ Model } & \multicolumn{4}{c|}{ PTPM } & \multicolumn{2}{c|}{ PTPM $_{\text {opt }}$} \\
Length & Time frame & States & Matches & Parsing (s) & Comp. (s) & States & Comp. (s) \\
\hline 200 & 101 & 20,602 & 5,050 & 0.01 & 15.31 & 515 & 0.24 \\
\hline 400 & 202 & 81,202 & 20,100 & 0.02 & 82.19 & 1,015 & 0.49 \\
\hline 600 & 301 & 181,802 & 45,150 & 0.03 & 236.80 & 1,515 & 0.71 \\
\hline 800 & 405 & 322,402 & 80,200 & 0.05 & 514.57 & 2,015 & 1.05 \\
\hline 1,000 & 503 & 503,002 & 125,250 & 0.06 & 940.74 & 2,515 & 1.24 \\
\hline
\end{tabular}

Table IV: Experiments: BLowUP

of symbolic matches in several dimensions, in just a few seconds. Another positive outcome is that PTPM is perfectly linear in the size of the input word for GEAR and ACCEL: this was expected as these examples are linear, in the sense that the number of states explored by PTPM is linear as these patterns feature no loops.

Note that the parsing time is not linear, but it could be highly improved: due to the relatively small size of the models usually treated by IMITATOR, this part was never properly optimized, and it contains several quadratic syntax checking functions.

The performances do not completely allow yet for an online usage in the current version of our algorithm and implementation (in [WHS17], we pushed the ACCEL case study for timed words of length up to 17,280,002). A possible direction is to perform and on-the-fly computation of the parametric zone graph, more precisely to do an onthe-fly parsing of the timed word automaton; this will allow
IMITATOR to keep in memory a single location at a time (instead of up to 25,137 in our experiments).

Finally, although this is not our original motivation, we believe that, if we are only interested in robust pattern matching, i.e., non-parametric pattern matching but with an allowed deviation ("guard enlargement") of the pattern automaton, then using the efficient 1-dimensional parameterized DBMs of [San15] would probably be an interesting alternative: indeed, in contrast to classical parameterized DBMs [HRSV02] (that are made of a matrix and a parametric polyhedron), the structure of [San15] only needs an $H \times H$ matrix with a single parameter, and seems particularly efficient.

\section{CONCLUSION}

We proposed a first approach to perform timed pattern matching in the presence of an uncertain specification. This allows us to synthesize parameter valuations and intervals 


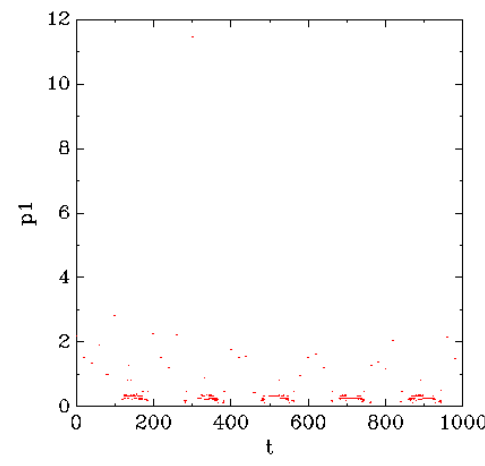

(a) Projection onto $t$ and $p$

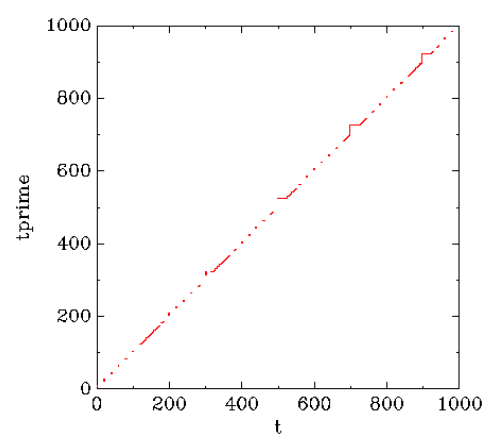

(b) Projection onto $t$ and $t^{\prime}$

Figure 6: Visualizing a large number of matches for GEAR $(|w=1467|)$

for which the specification holds on an input timed word. Our implementation using IMITATOR may not completely allow for online timed pattern matching yet, but already gives an interesting feedback in terms of parametric monitoring. Our second algorithm aiming at finding minimal or maximal parameter valuations is less sensitive to state space explosion. While our algorithms should be further optimized, we believe they pave the way for a more precise monitoring of real-time systems with an output richer than just timed intervals.

Future works: In [WHS17], we proposed an approach that can apply for online timed pattern matching. Its strength relied on the fact that an exhaustive search was not necessary, thanks to a skipping mechanism. Our next work will be to combine our current approach with the skipping mechanism of [WHS17].

Another challenge is the interpretation (and the visualization) of the results of parametric timed pattern matching. While the result of PTPM ${ }_{\mathrm{opt}}$ is natural, the fully symbolic result of PTPM remains a challenge to be interpreted; for example, the 125,250 matches for BLOWUP means the union of 125,250 polyhedra in 5 dimensions. We give a possible way to visualize such results in Fig. 6 for GEAR $(|w|=1,467)$ : in particular, observe in Fig. 6a that a single point exceeds 3 , only a few exceed 2 , while the wide majority remain in $[0,1]$. This helps to visualize how fast the gear is changed from 1 to 2 , and at what timestamps.

Also, exploiting the polarity of parameters, as done in [ADMN11] or in lower-bound/upper-bound parametric timed automata [HRSV02], may help to improve the efficiency of PTPM ${ }_{\text {opt }}$.

Finally, a natural extension of our work is to study monitoring using more expressive logics such as [BKMZ15].

\section{REFERENCES}

[ACEF09] Étienne André, Thomas Chatain, Emmanuelle Encrenaz, and Laurent Fribourg. An inverse method for parametric timed automata. International Journal of Foundations of Computer Science, 20(5):819-836, October 2009.

[AD94] Rajeev Alur and David L. Dill. A theory of timed automata. Theoretical Computer Science, 126(2):183235, April 1994.

[ADMN11] Eugene Asarin, Alexandre Donzé, Oded Maler, and Dejan Nickovic. Parametric identification of temporal properties. In $R V$, volume 7186 of $L N C S$, pages 147160. Springer, 2011.

[AFKS12] Étienne André, Laurent Fribourg, Ulrich Kühne, and Romain Soulat. IMITATOR 2.5: A tool for analyzing robustness in scheduling problems. In Dimitra Giannakopoulou and Dominique Méry, editors, FM, volume 7436 of $L N C S$, pages 33-36. Springer, August 2012.

[AHV93] Rajeev Alur, Thomas A. Henzinger, and Moshe Y. Vardi. Parametric real-time reasoning. In S. Rao Kosaraju, David S. Johnson, and Alok Aggarwal, editors, STOC, pages 592-601, New York, NY, USA, 1993. ACM.

[ALFS11] Yashwanth Annpureddy, Che Liu, Georgios E. Fainekos, and Sriram Sankaranarayanan. S-TaLiRo: A tool for temporal logic falsification for hybrid systems. In Parosh Aziz Abdulla and K. Rustan M. Leino, editors, TACAS, volume 6605 of $L N C S$, pages 254 257. Springer, 2011.

[And17] Étienne André. What's decidable about parametric timed automata? International Journal on Software Tools for Technology Transfer, 2017. To appear.

[BFM18] Alexey Bakhirkin, Thomas Ferrère, and Oded Maler. Efficient parametric identification for STL. In HSCC, pages 177-186. ACM, 2018.

[BFMU17] Alexey Bakhirkin, Thomas Ferrère, Oded Maler, and Dogan Ulus. On the quantitative semantics of regular expressions over real-valued signals. In Alessandro Abate and Gilles Geeraerts, editors, FORMATS, volume 10419 of LNCS, pages 189-206. Springer, 2017. 
[BHZ08] Roberto Bagnara, Patricia M. Hill, and Enea Zaffanella. The Parma Polyhedra Library: Toward a complete set of numerical abstractions for the analysis and verification of hardware and software systems. Science of Computer Programming, 72(1-2):3-21, 2008.

[BKMZ15] David A. Basin, Felix Klaedtke, Samuel Müller, and Eugen Zalinescu. Monitoring metric first-order temporal properties. Journal of the ACM, 62(2):15:115:45, 2015.

[BY03] Johan Bengtsson and Wang Yi. Timed automata: Semantics, algorithms and tools. In Jörg Desel, Wolfgang Reisig, and Grzegorz Rozenberg, editors, Lectures on Concurrency and Petri Nets, Advances in Petri Nets, volume 3098 of LNCS, pages 87-124. Springer, 2003.

[CJL17] Franck Cassez, Peter Gjøl Jensen, and Kim Guldstrand Larsen. Refinement of trace abstraction for real-time programs. In Matthew Hague and Igor Potapov, editors, $R P$, volume 10506 of $L N C S$, pages 42-58. Springer, 2017.

[FJS07] Frantisek Franek, Christopher G. Jennings, and William F. Smyth. A simple fast hybrid patternmatching algorithm. Journal of Discrete Algorithms, 5(4):682-695, 2007.

[FR08] François Fages and Aurélien Rizk. On temporal logic constraint solving for analyzing numerical data time series. Theoretical Computer Science, 408(1):55-65, 2008.

[HAF14] Bardh Hoxha, Houssam Abbas, and Georgios E. Fainekos. Benchmarks for temporal logic requirements for automotive systems. In Goran Frehse and Matthias Althoff, editors, ARCH@CPSWeek, volume 34 of EPiC Series in Computing, pages 25-30. EasyChair, 2014.

[HOW14] Hsi-Ming Ho, Joël Ouaknine, and James Worrell. Online monitoring of metric temporal logic. In Borzoo Bonakdarpour and Scott A. Smolka, editors, $R V$, volume 8734 of $L N C S$, pages 178-192. Springer, 2014.

[HRSV02] Thomas Hune, Judi Romijn, Mariëlle Stoelinga, and Frits W. Vaandrager. Linear parametric model checking of timed automata. Journal of Logic and Algebraic Programming, 52-53:183-220, 2002.

[JLR15] Aleksandra Jovanović, Didier Lime, and Olivier H. Roux. Integer parameter synthesis for timed automata. IEEE Transactions on Software Engineering, 41(5):445-461, 2015.

[KCDK15] Aaron Kane, Omar Chowdhury, Anupam Datta, and Philip Koopman. A case study on runtime monitoring of an autonomous research vehicle (ARV) system. In Ezio Bartocci and Rupak Majumdar, editors, $R V$, volume 9333 of $L N C S$, pages 102-117. Springer, 2015 .
[Koy90] Ron Koymans. Specifying real-time properties with metric temporal logic. Real-Time Systems, 2(4):255299, 1990.

[Mil00] Joseph S. Miller. Decidability and complexity results for timed automata and semi-linear hybrid automata. In Nancy A. Lynch and Bruce H. Krogh, editors, HSCC, volume 1790 of LNCS, pages 296309. Springer, 2000.

[RFB14] Thomas Reinbacher, Matthias Függer, and Jörg Brauer. Runtime verification of embedded realtime systems. Formal Methods in System Design, 44(3):203-239, 2014.

[San15] Ocan Sankur. Symbolic quantitative robustness analysis of timed automata. In Christel Baier and Cesare Tinelli, editors, TACAS, volume 9035 of $L N C S$, pages 484-498. Springer, 2015.

[UFAM14] Dogan Ulus, Thomas Ferrère, Eugene Asarin, and Oded Maler. Timed pattern matching. In Axel Legay and Marius Bozga, editors, FORMATS, volume 8711 of LNCS, pages 222-236. Springer, 2014.

[UFAM16] Dogan Ulus, Thomas Ferrère, Eugene Asarin, and Oded Maler. Online timed pattern matching using derivatives. In Marsha Chechik and Jean-François Raskin, editors, TACAS, volume 9636 of $L N C S$, pages 736-751. Springer, 2016.

[Ulu17] Dogan Ulus. Montre: A tool for monitoring timed regular expressions. In Rupak Majumdar and Viktor Kuncak, editors, CAV, Part I, volume 10426 of LNCS, pages 329-335. Springer, 2017.

[WAH16] Masaki Waga, Takumi Akazaki, and Ichiro Hasuo. A Boyer-Moore type algorithm for timed pattern matching. In Martin Fränzle and Nicolas Markey, editors, FORMATS, volume 9884 of LNCS, pages 121-139. Springer, 2016.

[WHS17] Masaki Waga, Ichiro Hasuo, and Kohei Suenaga. Efficient online timed pattern matching by automatabased skipping. In Alessandro Abate and Gilles Geeraerts, editors, FORMATS, volume 10419 of LNCS, pages 224-243. Springer, 2017.

\section{APPENDIX}

\section{A. Handling thousands of polyhedral disjuncts}

IMITATOR handles non-convex constraints (finite unions of polyhedra); while most case studies solved by IMITATOR in the past handle simple constraints (made of a few disjuncts), the experiments in this manuscript may handle up to dozens of thousands of such polyhedra. We therefore had to disable an inclusion test of a newly computed state into the already computed constraint: this test usually has a very interesting gain but, on our complex polyhedra, it had disastrous impact on the performance, due to the inclusion check of a (simple) new convex polyhedron into a disjunction of dozens of thousands of convex polyhedra. To disable this check, we added a new option (not set by 
default) to the master branch of IMITATOR, and used it in all our experiments.

\section{B. Significantly decreasing IMITATOR computation time}

In a preliminary version of our experiments, we obtained a computation time of up to 71 times slower than in the current version (e.g., 1201.2 instead of 16.90 for PTPM applied to ACCEL with an input word of size 25,137). It turned out that a frequent manual invocation of the OCaml garbage collector was responsible for this much slower time. Removing this invocation made our experiments much faster, while keeping the usual speed for the collection of benchmarks of IMITATOR. Recall that the experiments in this paper are unusual as the size of the input model (in terms of number of locations) is an order of magnitude larger than IMITATOR's historical benchmarks. 